\title{
Plan S y ecosistema de revistas españolas de ciencias sociales hacia el acceso abierto: amenazas y oportunidades
}

\author{
Plan $S$ and ecosystem of Spanish journals of \\ social sciences towards open access: threats and \\ opportunities
}

\author{
Alexandre López-Borrull; Candela Ollé-Castellà; Francesc García-Grimau; \\ Ernest Abadal
}

Cómo citar este artículo:

López-Borrull, Alexandre; Ollé-Castellà, Candela; García-Grimau, Francesc; Abadal, Ernest (2020). "Plan S y ecosistema de revistas españolas de ciencias sociales hacia el acceso abierto: amenazas y oportunidades". El profesional de la información, v. 29, n. 2, e290214.

https://doi.org/10.3145/epi.2020.mar.14

Artículo recibido el 07-08-2019 Aceptación definitiva: 17-12-2019

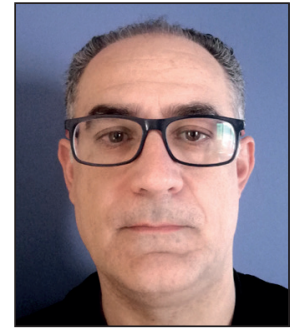

Alexandre López-Borrull $\square$ https://orcid.org/0000-0003-1609-2088

Universitat Oberta de Catalunya Estudis de Ciències de la Informació i la Comunicació

Av. Tibidabo, 39-43

08035 Barcelona, España

alopezbo@uoc.edu

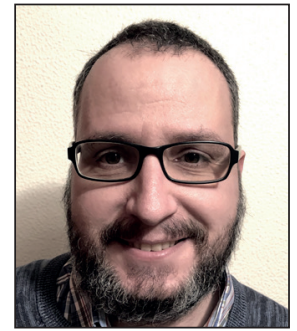

Francesc García-Grimau

https://orcid.org/0000-0001-8969-7868

Universitat de Barcelona

Fac. d'Informació i Mitjans Audiovisuals

Melcior de Palau, 140

08014 Barcelona, España

francesc.garcia.@ub.edu

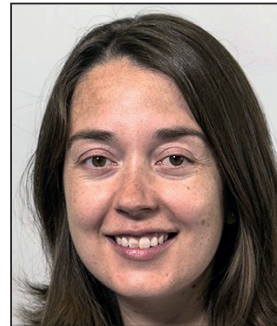

Candela Ollé-Castellà

https://orcid.org/0000-0002-8302-4790

Universitat Oberta de Catalunya

Estudis de Ciències de la Informació i la

Comunicació

Av. Tibidabo, 39-43

08035 Barcelona, España

collec@uoc.edu

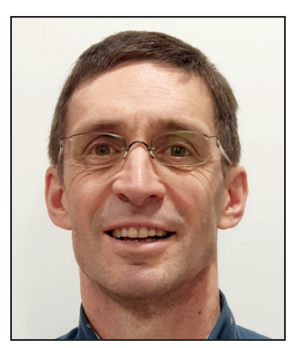

\section{Resumen}

El Plan S promueve que todos los artículos derivados de investigaciones financiadas por las agencias que lo suscriben sean puestos en acceso abierto de forma inmediata, ya sea en revistas o en repositorios que cumplan con determinadas condiciones, a partir de enero de 2021. Ha sido promovido por Science Europe, una agrupación de agencias de financiación pública de la investigación a través de la cOAlition $S$, y ha publicado dos versiones que han generado un intenso debate académico en todo el mundo. Aunque ninguna agencia de investigación pública o privada del estado español se ha comprometido de momento a apoyar el Plan S, es interesante valorar como dicho Plan puede afectar al ecosistema de revistas científicas españolas. Se presentan los resultados del análisis de las revistas españolas de ciencias sociales que figuran en el SCImago Journal Rank y el cumplimiento que tienen de los requisitos que la cOAlition S ha descrito para que las revistas sean consideradas de acceso abierto de calidad. Dichos requisitos están relacionados con la apertura de los contenidos, el modelo de pago, aspectos técnicos y los derechos de autor. Se concluye que, aunque la mayor parte

Agradecimientos

Este estudio se ha realizado en el marco del proyecto Ciencia abierta en España (RTI2018-094360-B-I00) financiado por el Programa Estatal de I+D+I del Ministerio de Ciencia, Innovación y Universidades. 
de las revistas cumplen los requisitos relativos a los contenidos abiertos, les quedan como retos los aspectos técnicos de máxima interoperabilidad y reutilización. Se recomienda a las revistas que tomen el Plan S como oportunidad y aprovechen para llevar a cabo una transformación digital plena.

\title{
Palabras clave
}

Plan S; Revistas científicas; Ciencias sociales; Comunicación científica; Publicación académica; Acceso abierto; APC; Ciencia abierta; Derechos de autor; España.

\begin{abstract}
Plan $S$ promotes that all articles from research funded by public financing agencies that subscribe it, have to be publicly available immediately, either in open access journals or in repositories that meet certain conditions, as of January 2021. Plan $S$ has been promoted by Science Europe, a group of public research funding agencies through the cOAlition $S$, and has published two versions that have generated intense academic debate worldwide. Even though no public or private research agency of Spain has been committed at the moment to support Plan S, it is interesting to assess how this Plan can affect the ecosystem of Spanish scientific journals. We present the results of the analysis of the Spanish journals of SCImago Journal Rank in Social Sciences and the compliance that they have with the requirements that cOAlition $S$ has described for the journals to be considered of Open Access of quality. These requirements are related to the opening of the content, the payment model, technical aspects and copyright. It is concluded that although most journals meet the requirements related to open content, they are left with challenges related to the technical aspects of maximum interoperability and reuse. Journals are recommended to take the Plan $S$ as an opportunity and take advantage to carry out a full digital transformation.
\end{abstract}

\section{Keywords}

Plan S; Scholarly journals; Social sciences; Academic publication; Scholarly communication; Open access; Open science; Copyright; Spain.

\section{Introducción}

La ruta hacia el pleno acceso abierto al conocimiento científico ha estado marcada por un trabajo paralelo entre las primeras declaraciones coordinadas de científicos y el trabajo lento pero directo de las políticas institucionales, sobre todo en el caso de la Comisión Europea. Si en un principio el acceso abierto era la parada final, ahora queda claro que es una estación más hacia el nuevo paradigma de la ciencia abierta (Anglada; Abadal, 2018). En este camino se aprecia un equilibrio claro entre el activismo proselitista (Melero, 2005) y el consenso paciente a través de múltiples informes y posicionamientos públicos (Scheliga; Friesike, 2014; Comisión Europea, 2016; Ayris et al., 2018).

El crecimiento del acceso abierto en la producción científica se ha ido incrementando en los últimos años (Piwowar et al., 2018; Science Metrix, 2018) y en estos momentos existen pocas dudas del cambio de modelo que se está produciendo en la comunicación científica. Son muchos los autores que consideran que la transición ya está prácticamente concluida y que actualmente la pregunta a formular ya no es lo que va a pasar (¿será el acceso abierto el nuevo modelo de comunicación científica?) sino cómo y cuándo pasará (Pinfield, 2015).

La propia Comisión Europea también lo considera de esta forma. De hecho, en la nueva versión de las Recomendaciones sobre acceso y preservación de la información científica (Comisión Europea, 2018), ya indica que todas las publicaciones derivadas de proyectos con financiación pública deberán estar en acceso abierto en 2020 como muy tarde. De cualquier forma está claro que sin controles de seguimiento ni establecimiento de incentivos, va a ser difícil que se cumplan estas obligaciones. Los últimos estudios sobre proporciones de acceso abierto (con variaciones según la disciplina o el país), en general superan por poco la mitad de la producción científica actual (Science Metrix, 2018).

En este contexto de final de una época de transición y de constatación de las dificultades para culminar el proceso hacia la total disponibilidad en abierto de la producción científica aparece el Plan S, que tiene por objetivo actuar de catalizador y acelerar la transición. El Plan $S$ se publicó el 4 de septiembre de 2018 (y fue actualizado el 31 de mayo del 2019) por un grupo de organizaciones estatales de financiación de la investigación, con el apoyo de la Comisión Europea y el Consejo Europeo de Investigación (ERC), bajo el nombre de cOAlition S (cOAlition S, 2019a). El Plan S tiene por objetivo conseguir que a partir del 1 de enero de 2021 todas las publicaciones -con un claro énfasis en las revisadas por pares- financiadas con fondos públicos estén publicadas o disponibles después de su publicación en revistas, repositorios o plataformas de acceso abierto. Dicho plan consta de un principio general:

“Con efecto a partir de 2021, todas las publicaciones académicas sobre los resultados de investigaciones financiadas por subvenciones públicas o privadas proporcionadas por consejos de investigación y organismos de financiación nacionales, regionales e internacionales deben publicarse en revistas de acceso abierto, en plataformas de acceso abierto, o ponerse a disposición de inmediato a través de repositorios de acceso abierto sin embargo". 
Añade 10 principios complementarios para que el principal pueda tener sentido:

1. los autores deben conservar los derechos de autor de sus publicaciones, que deben publicarse bajo una licencia abierta como Creative Commons (CC BY);

2. los miembros de la coalición deben establecer criterios y requisitos sólidos para las revistas y plataformas de acceso abierto que cumplan con los requisitos;

3. también deberían ofrecer incentivos para la creación de revistas y plataformas de acceso abierto compatibles, si aún no existen;

4. las tasas de publicación deben ser sufragadas por los financiadores o las universidades, no por investigadores individuales;

5. las tasas de publicación deben ser estandarizadas y limitadas;

6. las universidades, las organizaciones de investigación y las bibliotecas deben alinear sus políticas y estrategias;

7. el plazo para los libros y monografías podrá ampliarse más allá de 2020;

8. Ios archivos y repositorios abiertos deben ser reconocidos por su importancia;

9. las revistas híbridas de acceso abierto no cumplen con el principio clave;

10. los miembros de la coalición deben supervisar y sancionar el cumplimiento de este Plan.

Una de las debilidades del documento es su ambigüedad en algunos puntos, lo que permite diferentes interpretaciones y ha generado centenares de comentarios a varios aspectos especialmente complicados y/o conflictivos, como por ejemplo que el Plan $S$ establece incentivos para crear revistas/plataformas de acceso abierto o para convertir revistas existentes a un modelo de acceso abierto (University of Cambridge, 2019; Schiltz, 2018). Después de la publicación de la primera versión hubo un debate más o menos intenso, incluyendo una carta abierta firmada por más de 1.700 investigadores críticos con el Plan S (Plan S open letter, 2018); el posicionamiento a favor del Plan S por parte de China (Schiermeier, 2018); editoriales de algunas revistas criticando el Plan S como imperialismo académico (Burgman, 2018); o bien reflexiones sobre si el Plan S es exportable a América Latina o el resto del Global South (Poynder, 2019). Cabe destacar la visión de Amelica, estrategia propia de América Latina para llegar al objetivo común del acceso abierto (Amelica, 2019; Becerril-García, 2019).

Uno de los aspectos que generó cierto debate fue si $D O A J$ debería ser la lista blanca de las revistas que cumplían con el Plan S o debían ser los certificadores del cumplimiento (Strinzel et al., 2019). Este debate hizo que DOAJ hiciera una declaración de su posicionamiento, a la vez que aclaraba que estar en DOAJ no equivale a cumplir el Plan S, que no todas las revistas que pueden ahora mismo cumplir el Plan $S$ están en $D O A J$, y que trabajarán para que ambos grupos queden claros y diferenciados (DOAJ, 2019).

En la segunda versión, la cOAlition $S$ ha intentado concretar y suavizar algunos de los aspectos que generaban más dudas y debate, como por ejemplo la obligación inicial de una licencia CC BY 4.0 a la cual se han añadido excepciones para aceptar la licencia CC BY-SA 4.0 (share alike); o bien que los financiadores en sus programas puedan usar la CC-BY ND (no derivatives). También pasó de obligatoria a opcional la posibilidad de descarga del artículo en JATS XML, y en los principios se apostó más claramente por el espíritu de la declaración DORA (2012) en lo referente a la evaluación (cOAlition S, 2019b; 2019c). Varios textos han descrito los objetivos, estructura y repercusiones del Plan S y en ellos se puede ampliar esta información (Abadal et al., 2019; Bernal, 2018; López-Borrull, 2019; Martínez-Galindo et al., 2019; Sánchez-García, 2019).

Como era de imaginar el debate no sólo ha tenido lugar en los países firmantes del Plan S. En España, el 10 de octubre de 2018 se presentaba en el Congreso de los Diputados la proposición para debatir y aprobar la incorporación de España a la iniciativa cOAlition S en la Comisión de Ciencia, Innovación y Universidades. Durante la sesión se instó al Gobierno a llevar a cabo las acciones oportunas para la incorporación de la Agencia Estatal de Investigación como principal agente financiador del Sistema Nacional de I+D+i a la cOAlition $S$.

También se pedía la adopción de medidas oportunas para favorecer por parte de la Agencia Estatal de Investigación y de todos los agentes públicos de financiación del sistema español de I+D+i los principios del Plan S y su inclusión en el Plan Estatal de Investigación Científica y Técnica y de Innovación 2017-2020. La propuesta se modificó un mes después, incluyendo la necesidad de elaborar un informe con carácter previo al posible apoyo del Gobierno, que analice el impacto de la aplicación del Plan $S$ en la calidad y la competitividad de las publicaciones, en la libre elección de los investigadores cuando publican y en los costes que supondría que las tasas de publicación se pagasen por las entidades financiadores o las universidades.

Evidentemente un cambio importante para España sería el pago centralizado de los article processing charges (APC), en lugar de que los investigadores las asuman directamente. Actualmente España negocia muy pocos recursos de forma consorciada a nivel estatal, por lo que conseguir un solo interlocutor resulta complicado.

La propuesta marca tres rutas de cumplimiento para los artículos, y estas especificaciones vinculadas al principio número 9 (en contra de las revistas híbridas) quisieron suavizar el debate generado:

- publicación en revistas y plataformas de acceso abierto;

- publicación en revistas de suscripción con el inmediato depósito en repositorios;

- publicación en revistas de suscripción híbridas que están en un proceso de transformación al acceso abierto. 
Los contratos de estos acuerdos de transformación de las revistas híbridas deben ser públicos (incluidos los costes) y no pueden durar más allá de 2023. Para complementar y facilitar la transición al nuevo sistema de investigación que quiere alcanzar el Plan S, el documento dispone de una guía para la implementación, con concreciones técnicas que han ampliado y detallado algunas especificaciones en la actualización de mayo de 2019, y que será el núcleo y análisis de esta investigación, y se desarrollará en los siguientes apartados. Estos requisitos son muy importantes dado que deben cumplirse por parte de las revistas y repositorios que quieran satisfacer las condiciones de las agencias de financiación.

Recientemente se han publicado dos estudios que ponen en relación las revistas científicas y el Plan S. Un informe del Institute for Scientific Information (ISI) (Quaderi et al., 2019) calcula el impacto y el alcance del Plan S a partir del análisis bibliométrico de las publicaciones que se verán afectadas, ya que proceden de proyectos financiados por agencias adheridas al Plan S. Se trata de 120.000 artículos publicados en 10.000 revistas (el 6,4\% de las publicaciones en WoS en 2017). El estudio muestra que de las 3.700 revistas que incluyen más de seis artículos afectados por el Plan S, sólo 500 (el 14\%) están indexadas en DOAJ.

Por su parte, el estudio de Frantsvåg y Strømme (2019) quiere analizar el grado de cumplimiento del Plan S por parte de las más de 12.000 revistas incluidas en DOAJ. Los datos técnicos no se extraen directamente de cada uno de los portales web de las revistas, sino que se utilizan los metadatos de DOAJ con lo cual no se pueden comprobar todos los requisitos del Plan S. A pesar de esta limitación, encuentran que tan sólo el 8,8\% de las revistas incluidas en DOAJ cumplen con los indicadores. Por ello finalizan el artículo con algunas recomendaciones dirigidas a cOAlition $S$ que sirvan para facilitar la transición de las revistas a las nuevas exigencias:

- invertir en infraestructura técnica que facilite a las revistas cumplir con los indicadores;

- establecer diferencias de cumplimiento para las revistas con o sin APC;

- crear servicios certificadores del cumplimiento de los requisitos.

Nuestro objetivo ha sido analizar la situación de las revistas científicas españolas de ciencias sociales en relación con los requisitos establecidos por el Plan $S$ para conocer su grado de cumplimiento y su posible utilización como canal de difusión de la investigación financiada por estas agencias. A diferencia de los dos estudios antes mencionados nos centramos en revistas españolas sin tener en cuenta si publican o no artículos del Plan $S$ y, por otro lado recogemos los datos directamente de los portales de las revistas por lo que podemos analizar todos los indicadores establecidos por el Plan S.

Analizamos la situación de las revistas científicas españolas de ciencias sociales en relación con los requisitos del Plan $S$ para conocer su grado de cumplimiento

\section{Metodología}

El estudio se ha centrado en las revistas españolas de ciencias sociales indexadas en Scopus. La lista se ha obtenido a partir del SCImago Journal Rank, limitando la búsqueda a "España" (en el campo "Country") y "Social Sciences" (en "Área temática"). En enero de 2019 el número de revistas era de 214, que es con las que se ha trabajado. Inicialmente se analizaron los requisitos del Plan S en su primera versión (septiembre de 2018), que equivalían a 16 indicadores de cumplimiento o no cumplimiento (1/0). El primer análisis se llevó a cabo entre febrero y mayo de 2019 por parte de cuatro evaluadores previo consenso de las limitaciones y eliminación de las ambigüedades de los criterios, en la línea de otros trabajos publicados (Frantsvåg; Strømme, 2019). El 31 de mayo de 2019 los requisitos fueron actualizados (tabla 1). Se llevó a cabo un segundo análisis revisando los nuevos criterios y se actualizaron los resultados durante julio de 2019.

Los criterios establecidos por el Plan S se muestran en la tabla 1.

https://www.coalition-s.org/principles-and-implementation

Por razones técnicas, el criterio recomendable 1.1.13 no se ha estudiado en una primera fase. Durante el estudio se distinguieron, como hace el Plan $S$, dos tipos de criterios para las revistas, 12 obligatorios (1.1.1-1.1.8; 1.2.1-1.2.4) y 6 opcionales (1.1.9-1.1.15).

El cumplimiento de los criterios ha sido evaluado en función de las siguientes fuentes de información:

- sitios web de las revistas científicas;

- instrucciones a los autores de artículos;

- descripción de la revista proporcionada por la propia editorial;

- sitio web Sherpa/Romeo y Dulcinea en caso de duda ante los criterios de cumplimiento de los derechos de autor;

- revisión y análisis de artículos concretos publicados en los últimos números de la revista.

Los datos del estudio pueden consultarse en: https://doi.org/10.6084/m9.figshare.9249911.v1

\subsection{Limitaciones del estudio}

Cabe destacar que algunos de los requisitos técnicos generaron dudas sobre la interpretación y cómo evaluarlos adecuadamente. Para ello se optó por una interpretación flexible y favorable a reconocer el cumplimiento, aunque no estuviera la información suficientemente descrita en la web de la revista. Ello muestra que en algunos casos podrán cumplirse algunos requisitos simplemente mejorando la descripción de políticas y estadísticas. 
Tabla 1. Descripción de los requisitos obligatorios y opcionales establecidos por el plan S para las revistas y plataformas de acceso abierto

\begin{tabular}{|c|c|}
\hline \multicolumn{2}{|c|}{ Condiciones obligatorias básicas } \\
\hline 1.1.1 & Estándares de revisión de calidad siguiendo el Committee on Publication Ethics (COPE) y otros \\
\hline 1.1 .2 & Descripción detallada de las políticas editoriales, con estadísticas anuales \\
\hline 1.1 .3 & Retención del copyright por parte de los autores \\
\hline 1.1 .4 & Permitir publicación inmediata en licencia abierta y permitir depósito repositorio \\
\hline \multicolumn{2}{|c|}{ Condiciones técnicas obligatorias } \\
\hline 1.1 .5 & Uso de identificadores persistentes como DOI (digital object identifier), URN (uniform resource name) o Handle \\
\hline 1.1 .6 & Depósito del contenido en entornos de preservación digital \\
\hline 1.1.7 & Metadatos de artículo de alta calidad, en formatos interoperables no propietarios, incluyendo financiación \\
\hline 1.1 .8 & Información legible por ordenador del estado OA y la licencia en el artículo \\
\hline \multicolumn{2}{|c|}{ Criterios altamente recomendables adicionales } \\
\hline 1.1 .9 & Apoyo a identificadores persistentes para autores y entidades como Orcid \\
\hline 1.1 .10 & Registro de la política de autoarchivo en Sherpa/Romeo \\
\hline 1.1 .11 & Permitir descarga del texto completo en formatos estándares legibles por ordenador tales como JATS XML \\
\hline 1.1 .12 & Depósito directo de la publicación por la editorial a un repositorio OA Plan S \\
\hline 1.1 .13 & Metadatos conformes con OpenAIRE \\
\hline 1.1 .14 & Enlaces a datos, código y otros outputs en repositorios externos \\
\hline 1.1 .15 & Datos de citas abiertas de acuerdo con estándares Initiative for Open Citations (I4OC) \\
\hline \multicolumn{2}{|c|}{ 1.2 Condiciones específicas requeridas para revistas y plataformas de acceso abierto } \\
\hline 1.2 .1 & La revista / plataforma debe formar parte del Directory of Open Access Journals (DOAJ) o en proceso de ser registrado \\
\hline 1.2 .2 & No deben existir réplicas paralelas de pago \\
\hline 1.2 .3 & Coste y precio transparente \\
\hline 1.2 .4 & Exenciones y descuentos para autores de economías de bajos ingresos \\
\hline
\end{tabular}

\section{Resultados y discusión}

Se presentan los resultados del análisis aplicando la metodología presentada anteriormente. Se han analizado 214 revistas de ciencias sociales editadas en su mayoría por el sector público (universidades, CSIC, etc.).

\subsection{Requisitos obligatorios}

En primer lugar, en relación con una visión general de los requisitos obligatorios, se puede observar en la tabla 2 que existe una gran disparidad en cuanto al cumplimiento de cada uno de ellos. Mientras un grupo de requisitos (1.1.1, 1.1.5, 1.2.2-1.2.4) tienen un cumplimiento cercano o por encima del $70 \%$, cuatro indicadores son cumplidos por menos de un tercio de las revistas $(1.1 .2,1.1 .3,1.1 .6,1.1 .7)$.

Si nos remitimos al aspecto principal que ha llevado a la gestación del Plan, la publicación en abierto de forma inmediata de los artículos (1.1.4), vemos que lo cumplen cerca del $45 \%$ de las revistas, aunque el 54\% forman parte de DOAJ, lo que hace pensar que existe un $10 \%$ de revistas en acceso abierto que no tienen la licencia abierta que exige el Plan S. Cabe destacar que algunos de los requisitos, como el CC BY 4.0, se han incluido para que se permita utilizar futuras herramientas de valor añadido de minería de datos u otras bases de datos que pueden tener sentido comercial, aunque el artículo no lo tenga. Se valora la importancia futura de este aspecto porque, como en el caso de los datos de investigación, la reutilización es un sentido último para las instituciones europeas, tal y como se ha podido comprobar en la directiva europea que cita los datos de investigación (Comisión Europea, 2019). Así, en el blog de divulgación científica de Villatoro (2019) se expone el posible giro que la minería de artículos científicos puede dar a la investigación. Por otro lado, existen interesantes experimentos de utilización de minería de textos en la historia de la medicina (Thompson et al., 2016) o en la comparación de 15 millones de artículos científicos (Westergaard et al., 2018).

Por lo que respecta al mayoritario cumplimiento de los indicadores relacionados con el coste y el precio (1.2.2-

El requisito de publicación en abierto de forma inmediata de los artículos lo cumplen cerca del $45 \%$ de revistas, aunque el $54 \%$ forman parte de DOAJ, por lo que un $10 \%$ de revistas en OA no tienen la licencia abierta que exige el Plan $S$ 
1.2.4), se interpreta que muy pocas revistas son de pago para los autores, de forma que no necesitan disponer de los descuentos para autores de países en desarrollo. Tampoco es habitual la existencia de mirrors de pago paralelos, que desde el punto de vista del Plan $S$ se interpreta como una revista híbrida.

Tabla 2. Grado de cumplimiento de los requisitos obligatorios (ob) y opcionales (op) del Plan S por parte de las revistas españolas en CCSS.

\begin{tabular}{|c|c|c|c|}
\hline Indicador & Breve descripción & ob/op & Grado de cumplimiento (\%) \\
\hline 1.2 .2 & Sin mirror journals & $o b$ & 91,1 \\
\hline 1.2 .3 & Coste y precio transparente & $o b$ & 83,2 \\
\hline 1.1 .5 & Uso de identificadores persistentes DOI o handle & $o b$ & 69,6 \\
\hline 1.2 .4 & Exenciones y descuentos & $o b$ & 69,6 \\
\hline 1.1 .1 & Estándares de revisión de calidad & $o b$ & 69,2 \\
\hline 1.2 .1 & La revista / plataforma debe formar parte de DOAJ & $o b$ & 54,2 \\
\hline 1.1 .4 & Permitir publicación inmediata en licencia abierta & $o b$ & 44,9 \\
\hline 1.1 .8 & Información legible por ordenador del estado OA & $o b$ & 38,8 \\
\hline 1.1 .3 & Retención del copyright por los autores & $o b$ & 30,8 \\
\hline 1.1 .6 & Depósito en entornos de preservación digital & $o b$ & 27,1 \\
\hline 1.1 .10 & Registro política autoarchivo en Sherpa/Romeo & op & 23,8 \\
\hline 1.1 .9 & Apoyo a identificadores persistentes para autores y entidades & op & 22,4 \\
\hline 1.1.7 & Metadatos de artículo de alta calidad, en formatos interoperables & $o b$ & 22,0 \\
\hline 1.1 .2 & Descripción detallada de las políticas editoriales & ob & 15,9 \\
\hline 1.1 .12 & Depósito publicación por la editorial a un repositorio & op & 15,9 \\
\hline 1.1.14 & Enlaces a datos, código y otros en repositorios externos & op & 15,9 \\
\hline 1.1.11 & Permitir descarga del texto completo JATS XML & op & 11,7 \\
\hline 1.1 .15 & Datos de citas abiertas de acuerdo con estándares $140 C$ & op & 2,8 \\
\hline
\end{tabular}

\subsection{Requisitos opcionales}

Cuando el mismo análisis se lleva a cabo sobre los requisitos opcionales, vemos en la figura 1 que el cumplimiento es significativamente muy bajo. Ningún requisito supera el $25 \%$ de cumplimiento por parte de las revistas. Seguramente la dificultad general de cumplirlos ha hecho que se haya decidido por pasarlos a opcionales, pero algunos de ellos pueden ser claves en un futuro para permitir servicios de valor añadido. El uso de identificadores orcid, versiones en JATS XML, las políticas de autoarchivo en Sherpa/Romeo y el archivo en repositorios que cumplan el Plan $S$ son elementos que deberán acometer en un futuro. Se ha decidido que sean finalmente opcionales para favorecer la transición y el debate sobre el modelo de negocio híbrido.

Creemos significativo destacar también en este punto que, aunque hace años que los proyectos de financiación europeos empezaron a exigir la compartición de los datos de investigación, sólo un $16 \%$ de las revistas disponen de una política de compartición de datos, no ya en la propia revista, sino en repositorios externos (Pampel; Dallmaier-Tiessen, 2014).

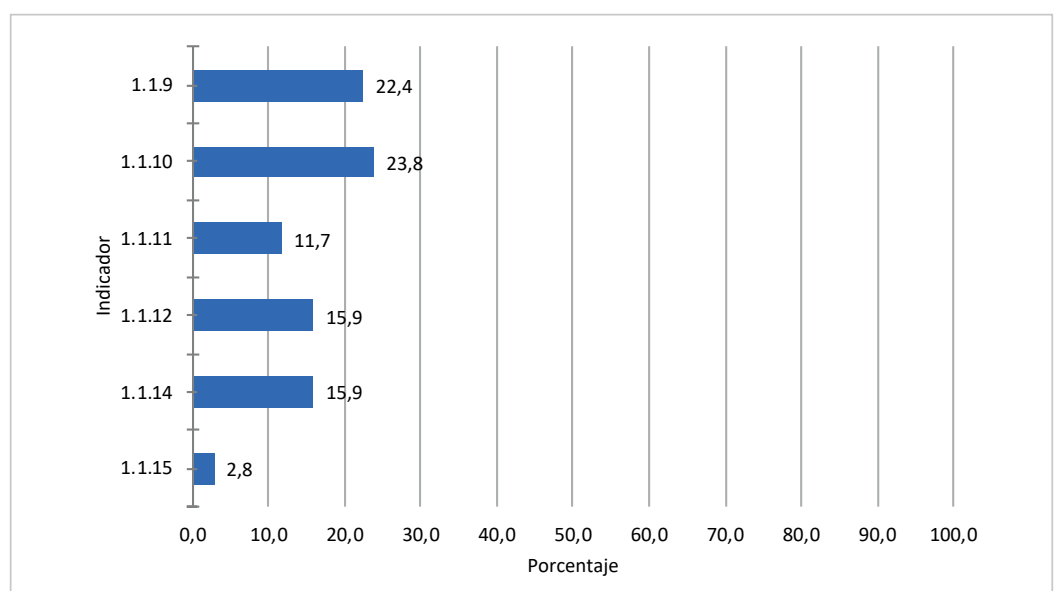

Figura 1. Porcentaje del grado de cumplimiento de los requisitos opcionales del Plan $S$ por parte de las revistas españolas en CCSS

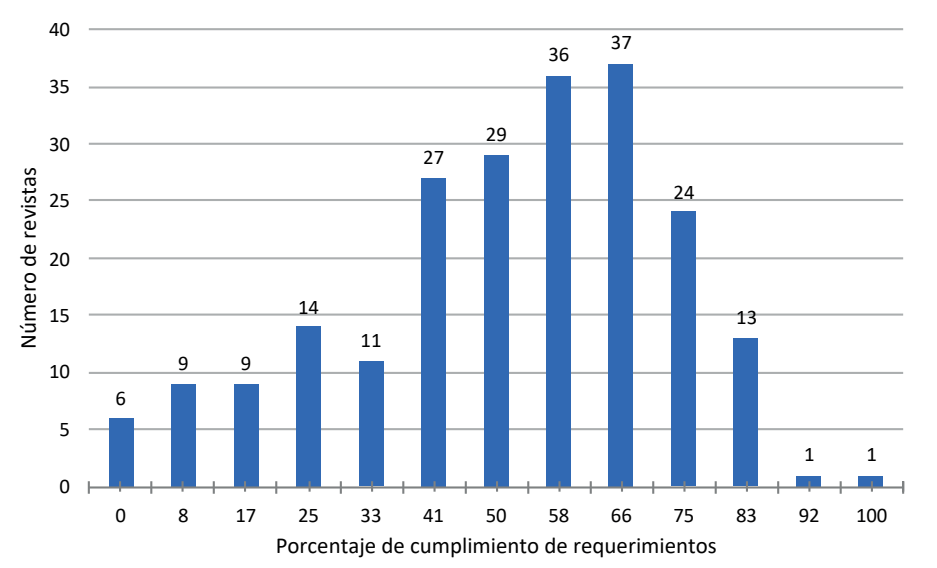

Figura 2. Distribución del cumplimiento de requisitos obligatorios por parte de las revistas 
Las figuras 2 (obligatorios) y 3 (opcionales) muestran el grado de cumplimiento de los indicadores por parte de las revistas. Se vislumbra un comportamiento en general asimétrico, y sólo dos de las revistas (Comunicar y Educación XXI) tienen un cumplimiento de 11 ó 12 de los 12 indicadores obligatorios. Aun así, el grueso de las revistas parece tener un cumplimiento medio de los requisitos. Además es de suponer que simplemente con una buena descripción de sus prácticas en sus webs podrían cumplir y mejorar entre dos y cuatro de los requisitos sin excesivos problemas. En lo que respecta a los requisitos opcionales, más del $50 \%$ de las revistas no cumplen ninguno, lo que muestra que son aspectos que quedan lejos de estar plenamente incorporados en el ecosistema estudiado.

También se ha podido observar en los foros habituales de debate cómo el impacto en el ecosistema científico de las revistas de momento ha sido menor, a la espera de lo que puedan llevar a cabo las agencias de financiación españolas tanto públicas como privadas. En este sentido se debe recordar que las fundaciones Bill \& Melinda Gates y Wellcome han asumido también el Plan $S$ para sus propios programas de financiación.

\subsection{Cumplimiento en relación con las editoriales, el cuartil y la temática}

En el trabajo de Frantsvåg y Strømme (2019) se encontró una relación directa entre revistas que hacen pagar APC y cumplimiento de los criterios, ya que el $25,6 \%$ de las revistas con APC los cumplían por tan sólo el 2,8 \% de las que no tienen APC. En lo que se refiere a las disciplinas, las humanidades y ciencias sociales tienen un menor índice de cumplimiento que el resto. En nuestro caso hemos analizado la relación con las editoriales, el cuartil y la temática.

Otro aspecto que se ha podido recoger en el estudio ha sido el papel de las universidades y el CSIC como editoriales relevantes en el ecosistema de revistas científicas en ciencias sociales. En la figura 4 se puede comprobar el cumplimiento promedio (recordemos, sobre 12 requisitos) de las revistas de las principales editoriales académicas. Cabe destacar la media del CSIC y de la Universitat Autònoma de Barcelona, que muestran un proceso de homogeneización y seguimiento de la calidad de las revistas elevado.

Por otra parte, también se quiso evaluar si el posicionamiento de las revistas en los cuartiles SJR presentaban algún tipo de patrón respecto al cumplimiento de los requisitos. Como se puede ver en la figura 5 , a mayor cuartil mayor cumplimiento. Más que entrar en una discusión sobre la calidad de unos u otros cuartiles, es probable que la competitividad añadida que exige intentar acceder y mantenerse lleva a cumplir algunos indicadores.

La figura 6 recoge el comportamiento

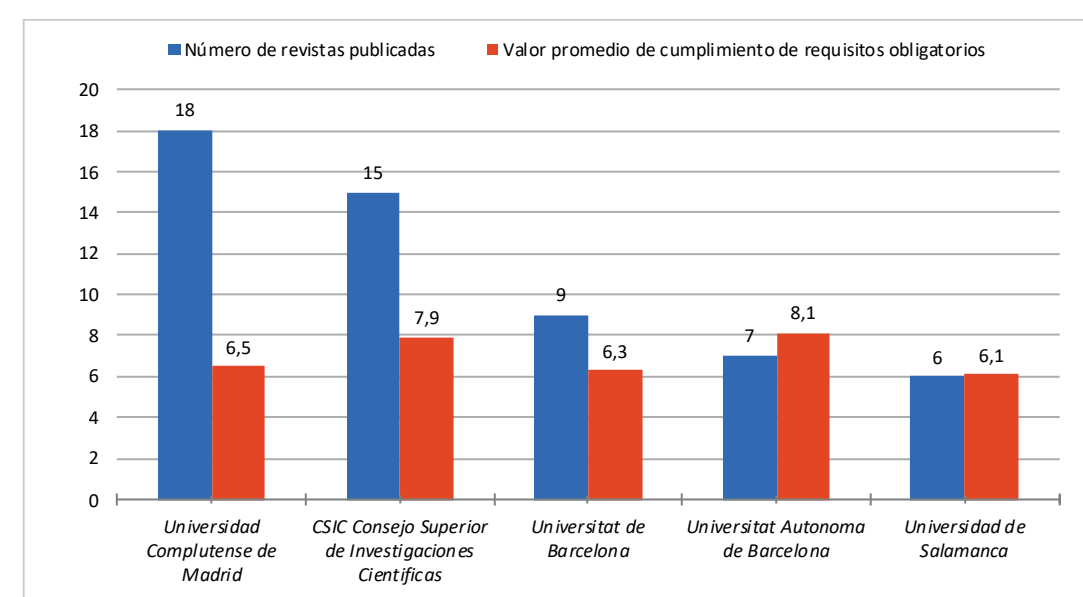

Figura 4. Promedio del cumplimiento de requisitos obligatorios por parte de las revistas de las principales editoriales de revistas españolas en CCSS

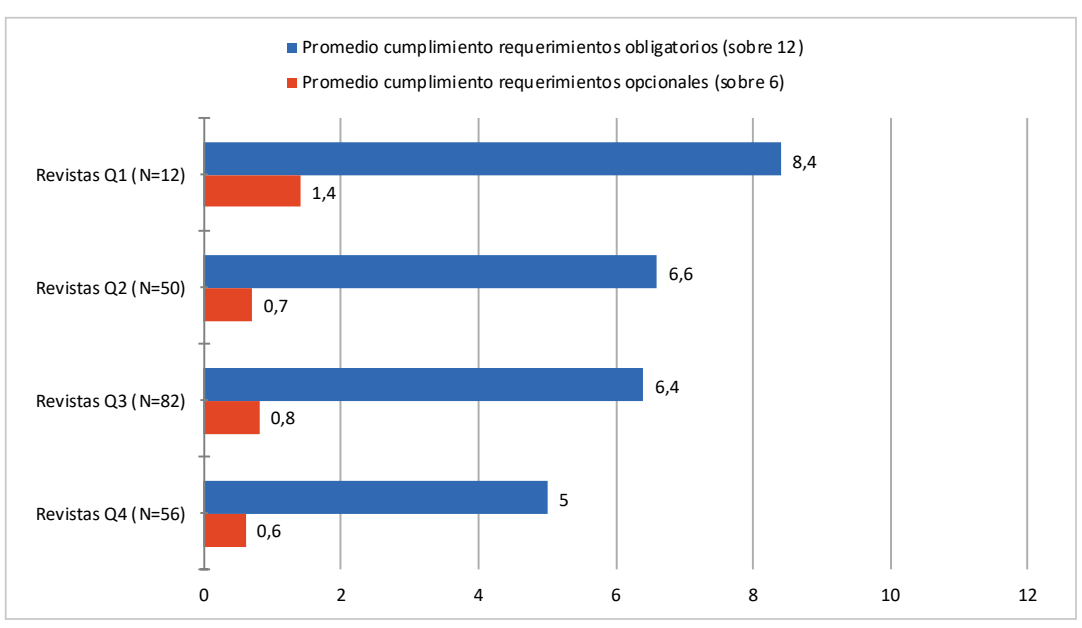

Figura 5. Promedio del cumplimiento de requisitos obligatorios por parte de las revistas en función del cuartil SJR 
de las revistas en función de las categorías temáticas $S J R$. Cabe recordar en este sentido, que dichas categorías temáticas no son unívocas, así que algunas revistas tienen $2,3,4$ y hasta 5 categorías, lo que hace más difícil la comparación ya que existe un solapamiento relativamente importante. Asimismo, el número de revistas en cada categoría también es diferente. Lo que sí parece observarse es un comportamiento diferencial en las categorías, con un mayor cumplimiento en Educación, Comunicación, Geografía y Ciencias Sociales (miscelánea) y uno menor en Ciencias Políticas y Derecho.

Finalmente, se consideró oportuna la concreción del cumplimiento de los requisitos de las revistas científicas en Comunicación (figura 7) y en Información y Documentación (figura 8), mostrándose también patrones asimétricos, es decir, algunas revistas como Comunicar, REDC y BiD cumplen casi todos los requisitos, mientras un grueso de revistas cumple cerca del $50 \%$ de los requisitos.

\section{Conclusiones y recomendaciones}

A continuación se presentan las principales conclusiones y recomendaciones del estudio realizado.

Por lo que respecta a los resultados generales, podemos afirmar que ninguno de los requisitos obligatorios $u$ opcionales es cumplido por el conjunto de las revistas científicas. Únicamente dos revistas, Educación XXI (12) y Comunicar (11) cumplen todos o casi todos los requisitos obligatorios, mientras que sólo 11 de las 214 revistas cumplen 10 de los 12 requisitos obligatorios que la cOAlition $S$ demanda a las revistas para que los investigadores puedan publicar en ellas.

Asimismo existe una correlación de cumplimiento de requisitos obligatorios con el cuartil que ocupan las revistas. A mayor cuartil, mayor cumplimiento de requisitos. Esta correlación es más atenuada en el caso de los opcionales. Por su parte, las grandes editoriales científicas de revistas españolas de ciencias sociales, de tipo académico, tienen un cumplimiento mayoritario de los requisitos y un diseño cada vez más homogéneo, considerando una

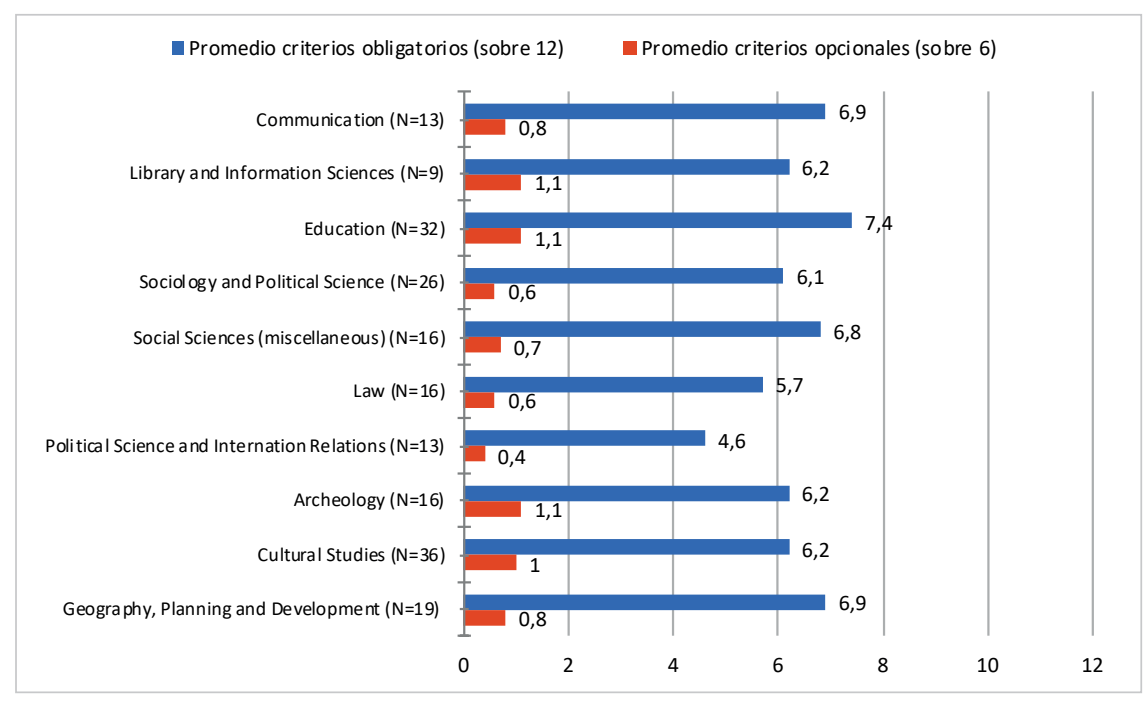

Figura 6. Promedio del cumplimiento de requisitos obligatorios y opcionales por categorías temáticas $S J R$ en ciencias sociales

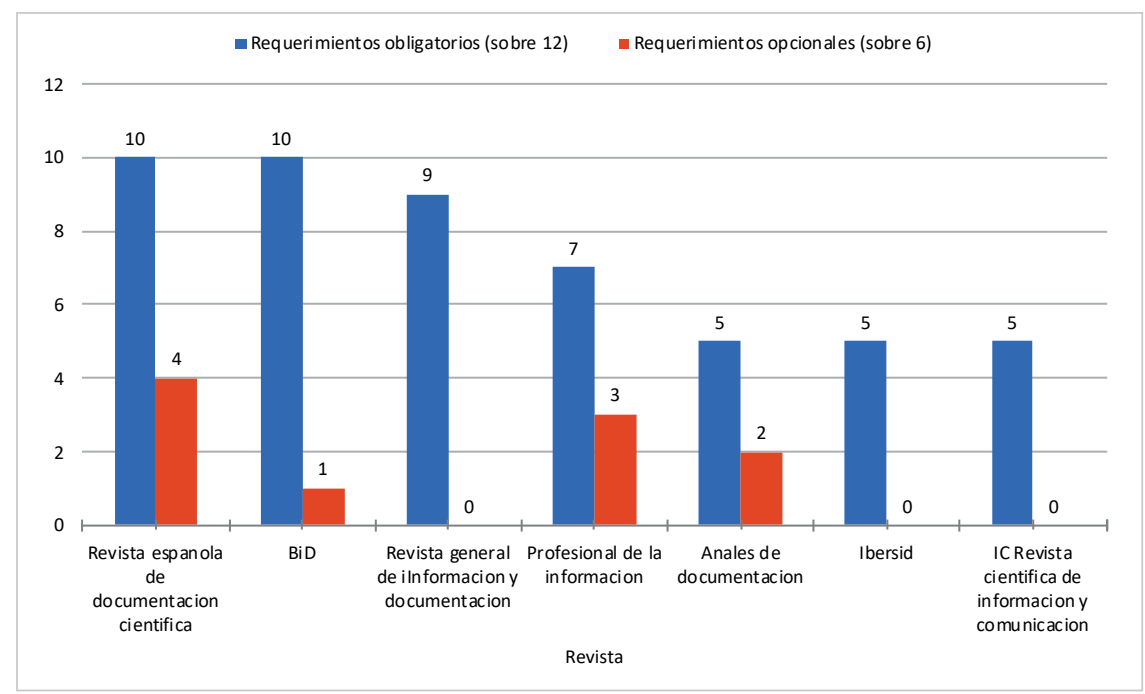

Figura 7. Cumplimiento de requisitos obligatorios y opcionales por parte de las revistas españolas en la categoría temática SJR Library and Information Science

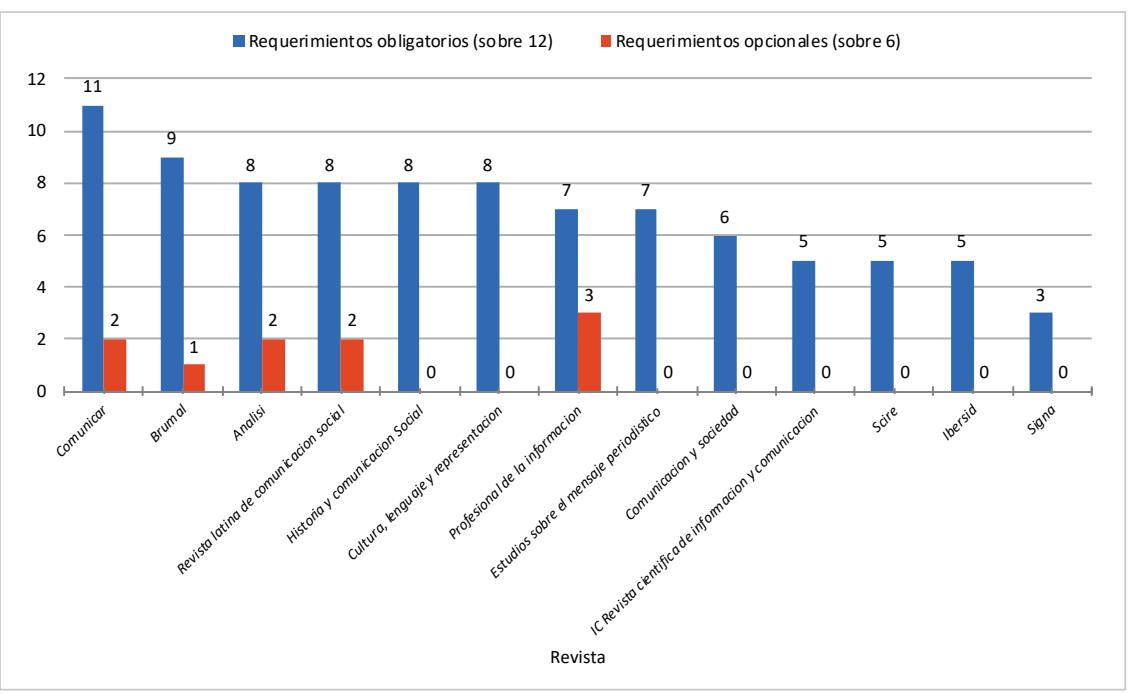

Figura 8. Cumplimiento de requisitos obligatorios y opcionales por parte de las revistas españolas en la categoría temática SJR Communication 
buena labor por parte de los servicios de publicación. Si consideramos las revistas por categorías temáticas, vemos que las revistas españolas de CCSS de las categorías temáticas de Library and Information Science y de Communication presentan una importante asimetría, con algunas revistas con un cumplimiento mayoritario de requisitos y un grupo de revistas que no llegan al 50\% de cumplimiento.
Ninguno de los requisitos obligatorios $u$ opcionales es cumplido por el conjunto de las revistas. Únicamente Educación XXI (12) y Comunicar (11) los cumplen todos o casi todos, y sólo 11 de las 214 revistas cumplen 10 de los 12 obligatorios

A tenor de los resultados anteriores, vemos que el Plan $S$ no es sólo una cuestión de acceso abierto, puesto que la mayor parte de las revistas científicas de ciencias sociales lo son (según $S J R, 123$ de las 214), sino que también intenta generar un cambio en las licencias y aspectos técnicos para tener un ecosistema de revistas científicas y plataformas de mejor calidad en acceso abierto.

Ello quiere decir que ahora mismo ninguno de los investigadores que a partir del 2021 tengan proyectos de investigación de alguna de las agencias de financiación que forman parte de la cOAlition S podría publicar en alguna de las 214 revistas españolas en ciencias sociales. Además, cabe destacar las sanciones que pueden recibir aquellos investigadores que no hagan caso a los requisitos de las agencias que forman parte de la cOAlition $S$, puesto que se crearán sistemas de monitorización.

En este sentido, hay que considerar que el Plan $S$ es también un conjunto de medidas que permiten a las revistas un ejercicio de mayor transparencia. Se ha podido comprobar que a menudo algunos de los criterios pueden cumplirse o no, pero el problema es que no están suficientemente explicitados. Como conclusión, el sistema científico español puede ser considerado sistémico por cantidad y calidad, así que no debería aislarse de las nuevas dinámicas y requisitos de publicación. La consideración del cumplimiento del Plan S como un sello de calidad en acceso abierto puede marcar la diferencia de cara el futuro, en un momento de crisis respecto al papel de las revistas y editoriales depredadoras.

En lo que respecta a recomendaciones a las revistas en función del estudio elaborado, creemos que las revistas españolas de CCSS deben tomar los requisitos del Plan $S$ como una oportunidad para hacer una definitiva transformación digital, la que no se hizo cuando se pasó a la gestión de artículos en formato pdf. Así, los requisitos son también una hoja de ruta de calidad y transparencia de las revistas científicas en acceso abierto.

Como se ha ido comentando a lo largo el artículo, en la mayor parte de las revistas científicas de CCSS, no es el modelo de negocio del acceso abierto o el ser revistas híbridas lo que impide que cumplan los requisitos, sino que son básicamente los requisitos técnicos. El cumplimiento del conjunto de criterios técnicos obligatorios y opcionales por parte de las revistas va a ser básico para poder desarrollar a su vez un sistema eficaz y eficiente de ciencia abierta, por cuanto aspectos como la preservación, el uso de indicadores, la compartición real de conjuntos de datos y la transparencia forman parte del espíritu del Plan S. Ello redunda en la importancia de poder formar parte cuanto antes del conjunto de revistas que cumplan con los requisitos.

Por nuestra parte, recomendamos encarecidamente a las revistas científicas que se evalúen internamente sobre el cumplimiento de los indicadores y que formen parte a su vez de su plan estratégico o de calidad. A veces, cumplir o no cumplir algunos de los requisitos, puede venir dado por acciones de comunicación de políticas, indicadores de calidad y estadísticas, sin requerir aportación extraordinaria de presupuesto.

En este sentido, en el caso de la aportación de presupuesto para el cumplimiento de algunos de los indicadores ya sean obligatorios $u$ opcionales, parece oportuno plantear un proceso de transformación digital de la revista o del conjunto de revistas de una misma editorial académica, que puede optimizar costes. En el caso de la publicación en JATS XML, metadatos de interoperabilidad de calidad, o las citas abiertas, cabe considerar que son requisitos que a corto o medio plazo otras agencias de evaluación de revistas científicas van a poder escoger. Así, ser de las primeras revistas en cumplir los requisitos del Plan $S$ puede significar un valor añadido diferencial respecto a otras muchas revistas científicas de impacto parecido. En un entorno competitivo como el actual puede tener el mismo valor que la difusión en la post publicación o bien la publicación de forma continua.

Como en otras etapas de la transición al pleno acceso abierto, siempre va a existir alguna excusa o bien algún colectivo reacio al cambio definitivo en la apuesta al acceso abierto, pero deben dotarse de pasarelas y transición que permita nivelar aquellas categorías temáticas con un número relevante de revistas híbridas, a la expectativa de que entre en un proceso de transformación o no.

Como oportunidades que se plantean en lo que respecta a las administraciones, el otro tipo de acción, a nivel de políticas de información, debería ser la realización de un informe que incluya el presupuesto necesario para la posible 
reconversión del ecosistema científico español de revistas, con los costes relacionados de la aplicación de los requisitos técnicos. Coincidimos con Frantsvåg y Strømme (2019) en que la administración pública debe invertir en el desarrollo de infraestructuras técnicas que faciliten a las revistas cumplir con los indicadores (por ejemplo, actualizar y mejorar las plataformas de edición de revistas). Es interesante tener en cuenta buenas prácticas como el mecanismo de financiación descrito en el artículo de De-Castro y Franck (2019) para revistas de acceso abierto no basadas en APCs. Esta idea debería ser coherente con el añadido en el principio 5 de la segunda versión del Plan $S$ donde se explicita que los financiadores apoyan la diversidad de modelos de negocio para revistas y plataformas de acceso abierto.

En el caso español debería analizarse si el portal Recyt y otros similares (como RACO, Redib) que están financiados con recursos públicos podrían ejercer esta función. De forma evidente, ello debería ir acompañado de una propuesta de adhesión al Plan $S$ o bien de creación de una política propia que fuera en la misma línea y con los objetivos de llegar al mismo punto final. Quizá es temprano para poder valorar si el Plan $S$ era el mejor de los planes posibles, pero aquellos que no se suman deberán explicitar cuál es su alternativa, en la línea del Plan $S$ o bien siguiendo la filosofía por la que opta Amelica. Cabría tener como ejemplo el liderazgo de UK Research Innovation que, a través del proyecto Society publishers accelerating open access and Plan S (SPA-OPS), ha marcado un análisis de cómo las sociedades editoras pueden hacer la transición hacia el pleno cumplimiento de los requisitos del Plan S (Wise; Estelle, 2019). Dicho informe de escenarios de futuro debería contemplar a su vez el coste de la posible creación de una plataforma científica que cumpliera con todos los requisitos del Plan $S$ a la vez que se apostara por vías alternativas de evaluación de la producción científica de la ciencia española.

En cuanto al futuro, se plantean dos tipos de acciones:

- a nivel académico, este mismo tipo de estudio debería llevarse a cabo en cada área temática, en especial en humanidades y en ciencia y tecnología;

- habría que evaluar los repositorios para comprobar también el cumplimiento de los requisitos del Plan S.

\section{Referencias}

Abadal, Ernest; López-Borrull, Alexandre; Ollé-Castellà, Candela; García-Grimau, Francesc (2019). “El Plan S para acelerar el acceso abierto: contexto, retos y debate generado". Hipertext.net, n. 19, pp. 75-83.

https://doi.org/10.31009/hipertext.net.2019.i19.06

AmeliCA (2019). Principios y valores. AmeliCA.

http://amelica.org/index.php/principios-y-valores

Anglada, Lluís; Abadal, Ernest (2018). “¿Qué es la ciencia abierta?”. Anuario ThinkEPI, v. 12, pp. $292-298$. https://doi.org/10.3145/thinkepi.2018.43

Ayris, Paul; López-De-San-Román, Alea; Maes, Katrien; Labastida, Ignasi (2018). Open science and its role in universities: A roadmap for cultural change. Leuven: LERU.

https://www.leru.org/files/LERU-AP24-Open-Science-full-paper.pdf

Becerril-García, Arianna (2019). “AmeliCA vs Plan S: mismo objetivo, dos estrategias distintas para lograr el acceso abierto". Blog Ameli, 10 enero.

http://amelica.org/index.php/2019/01/10/amelica-vs-plan-s-mismo-objetivo-dos-estrategias-distintas-para-lograr-elacceso-abierto

Bernal, Isabel (2018). "Plan S: Principios, hoja de ruta y reacciones". Enredadera, n. 32.

http://sitios.csic.es/web/enredadera/plan_s

Burgman, Mark (2018). “Open access and academic imperialism”. Conservation biology, v. 33, n. 1, pp. 5-6. https://doi.org/10.1111/cobi.13248

cOAlition S (2019a). Plan S. Making full and immediate open access a reality. European Science Foundation. https://www.coalition-s.org

cOAlition S (2019b). cOAlition S releases revised implementation guidance on Plan S following public feedback exercise. European Science Foundation.

https://www.coalition-s.org/revised-implementation-guidance

cOAlition S (2019c). Rationale for the revisions made to the Plan S principles and implementation guidance. European Science Foundation.

https://www.coalition-s.org/rationale-for-the-revisions 
Comisión Europea (2016). Open innovation. Open science. Open to the world. A vision for Europe. Directorate-General for Research and Innovation.

https://publications.europa.eu/en/publication-detail/-/publication/3213b335-1cbc-11e6-ba9a-01aa75ed71a1

Comisión Europea (2018). "Recomendación (UE) 2018/790 de la Comisión de 25 de abril de 2018 relativa al acceso a la información científica y a su preservación”. Diario oficial de la Unión Europea, L134/12, 31 mayo.

https://eur-lex.europa.eu/legal-content/ES/TXT/PDF/?uri=CELEX:32018H0790

Comisión Europea (2019). “Directiva (UE) 2019/1024 del Parlamento Europeo y del Consejo de 20 de junio de 2019 relativa a los datos abiertos y la reutilización de la información del sector público (versión refundida)". Diario oficial de la Unión Europea, L172/56, 26 junio.

https://eur-lex.europa.eu/legal-content/EN/TXT/PDF/?uri=uriserv:OJ.L_.2019.172.01.0056.01.SPA

De-Castro, Pablo; Franck, Gwen (2018). "Funding APCs from the research funder's seat: Findings from the EC FP7 Postgrant open access pilot". El profesional de la información, v. 28, n. 4, e280413.

https://doi.org/10.3145/epi.2019.jul.13

DOAJ (2019). DOAJ's open letter to SSHA communities about Plan S.

https://blog.doaj.org/tag/plan-s

DORA (2012). San Francisco declaration on research assessment. San Francisco: DORA.

https://sfdora.org/read

Frantsvåg, Jan-Eric; Strømme, Tormod-Eismann (2019). "Few open access journals are compliant with Plan S". Publications, v. 7, n. 2.

https://doi.org/10.3390/publications7020026

López-Borrull, Alexandre (2019). “Plan S: La velocidad del acceso abierto depende del punto de referencia?”. Comeln. Revista dels Estudis de Ciències de la Informació i la Comunicació, n. 84.

https://www.uoc.edu/divulgacio/comein/es/numero84/articles/plan-S-velocidad-acceso-abierto-punto-referencia.html

Martínez-Galindo, Francisco-Jesús; Rubio, Francisco; Hernández-San-Miguel, Javier; Fernández-Burguete, Sergio (2019). "Plan S: Challenges and opportunities in Spain". Insights, v. 32, n. 1, 17.

http://doi.org/10.1629/uksg.467

Melero, Remedios (2005). "Significado del acceso abierto (open access) a las publicaciones científicas: definición, recursos, copyright e impacto". El profesional de la información, v. 14, n. 4, pp. 255-266.

http://www.elprofesionaldelainformacion.com/contenidos/2005/julio/3.pdf

Pampel, Heinz; Dallmaier-Tiessen, Suenje (2014). "Open research data: From vision to practice”. In: Bartling, Sönke; Friesike, Sascha (eds.). Opening science. The evolving guide on how the internet is changing research, collaboration and scholarly publishing. Heidelberg: Springer, pp. 213-224. ISBN: 9783319000251

http://doi.org/10.1007/978-3-319-00026-8

Pinfield, Stephen (2015). "Making open access work: The 'state-of-the-art' in providing open access to scholarly literature". Online information review, v. 39, n. 5, pp. 604-636.

https://doi.org/10.1108/OIR-05-2015-0167

Piwowar, Heather; Priem, Jason; Larivière, Vincent; Alperin, Juan-Pablo; Matthias, Lisa; Norlander, Bree; Farley, Ashley; West, Jevin; Haustein, Stefanie (2018). "The state of OA: A large-scale analysis of the prevalence and impact of open access articles". PeerJ, n. 6, e4375.

https://doi.org/10.7717/peerj.4375

Plan S open letter (2018). Reaction of researchers to Plan S: Too far, too risky.

https://sites.google.com/view/Plansopenletter/open-letter

Poynder, Richard (2019). Plan S: What strategy now for the Global South?

https://richardpoynder.co.uk/Plan_S.pdf

Quaderi, Nandita; Hardcastle, James; Petrou, Christos; Szomszoret, Martin (2019). The Plan S footprint: Implications for the scholarly publishing landscape. ISI (Institute for Scientific Information); Web of Science Group.

https://clarivate.com/g/plan-s-footprint

Sánchez-García, Sandra (2019). “¿Estamos preparados para hacer frente a las exigencias del Plan S?”. Aula magna 2.0, 12 julio.

https://cuedespyd.hypotheses.org/6417

Scheliga, Kaja; Friesike, Sascha (2014). “Putting open science into practice: A social dilemma?”. First Monday, v. 19, n. 9. https://doi.org/10.5210/fm.v19i9.5381 
Schiermeier, Quirin (2018). “China backs bold plan to tear down journal paywalls”. Nature, n. 564, pp. 171-172. https://doi.org/10.1038/d41586-018-07659-5

Schiltz, Marc (2018). "Science without publication paywalls: cOAlition S for the realisation of full and immediate open access". PLoS medicine, v. 15, n. 9, e1002663.

https://doi.org/10.1371/journal.pmed.1002663

Science Metrix (2018). Analytical support for bibliometrics indicators: Open access availability of scientific publications. Final report.

http://www.science-metrix.com/sites/default/files/science-metrix/publications/science-metrix_open_access_ availability_scientific_publications_report.pdf

Strinzel, Michaela; Severin, Anna; Milzow, Katrin; Egger, Matthias (2019). "Blacklists and whitelists to tackle predatory publishing: A cross-sectional comparison and thematic analysis". mBio, v. 10, e00411-19.

https://doi.org/10.1128/mBio.00411-19

Thompson, Paul; Batista-Navarro, Riza-Theresa; Kontonatsios, Georgios; Carter, Jacob; Toon, Elizabeth; McNaught, John; Timmermann, Carsten; Worboys, Michael; Ananiadou, Sophia (2016). "Text mining the history of medicine". PLoS one, v. 11, n. 1, e0144717.

https://doi.org/10.1371/journal.pone.0144717

University of Cambridge (2019). Plan S - links, commentary and news items. University of Cambridge. Office of Scholarly Communication.

https://unlockingresearch-blog.lib.cam.ac.uk/?p=2433

Villatoro, Francisco R. (2019). "El descubrimiento científico usando minería de artículos científicos". La ciencia de la mula Francis, 4 julio.

https://francis.naukas.com/2019/07/04/el-descubrimiento-cientifico-usando-mineria-de-articulos-cientificos

Westergaard, David; Stærfeldt, Hans-Henrik; Tønsberg, Christian; Jensen, Lars-Juhl; Brunak, Søren (2018). "A comprehensive and quantitative comparison of text-mining in 15 million full-text articles versus their corresponding abstracts". PLoS computational biology, v. 14, n. 2, e1005962.

https://doi.org/10.1371/journal.pcbi.1005962

Wise, Alicia; Estelle, Lorraine (2019). Society publishers accelerating open access and Plan S (SPA-OPS) project. Figshare. Collection.

https://doi.org/10.6084/m9.figshare.c.4561397.v2

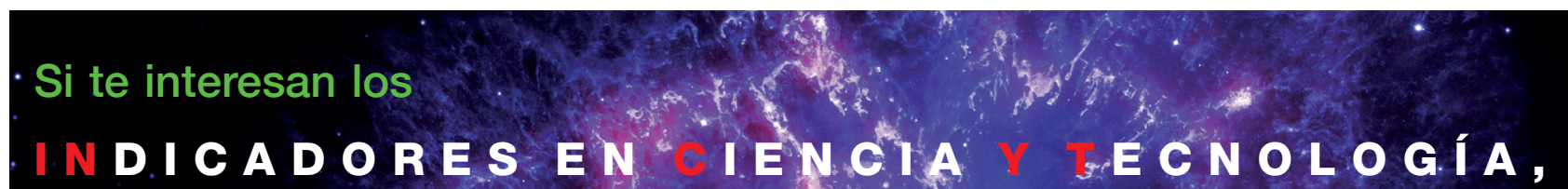

y todos los temas relaciona dos con lismédición de la ciểria, tales como:

Análisis de citás, Normalización de inompreś e instituciones: Impacto de

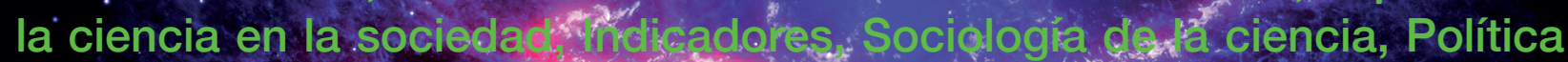

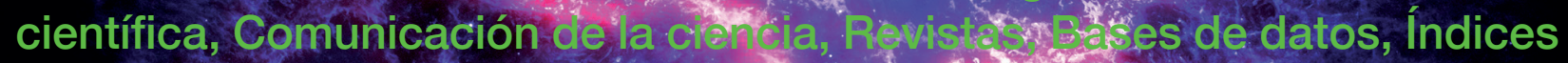

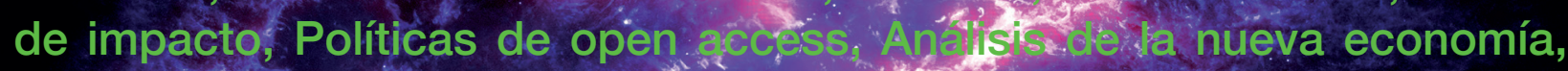
Mujer y ciencia, etc.

\section{Entonces es tu lista. Suscríbete en:}

http://whw.rediris.es/list/info/incyt.htmI 